\title{
Major role for mRNA binding and restructuring in sRNA recruitment by $\mathrm{Hfq}$
}

\author{
TOBY J. SOPER, ${ }^{1}$ KEVIN DOXZEN, $^{2}$ and SARAH A. WOODSON ${ }^{1,2,3}$ \\ ${ }^{1}$ Cell, Molecular and Developmental Biology and Biophysics Program, Johns Hopkins University, Baltimore, Maryland 21218, USA \\ ${ }^{2}$ T.C. Jenkins Department of Biophysics, Johns Hopkins University, Baltimore, Maryland 21218, USA
}

\begin{abstract}
Bacterial small RNAs (sRNAs) modulate gene expression by base-pairing with target mRNAs. Many sRNAs require the Sm-like RNA binding protein $\mathrm{Hfq}$ as a cofactor. Well-characterized interactions between DsrA sRNA and the rpoS mRNA leader were used to understand how $\mathrm{Hfq}$ stimulates sRNA pairing with target mRNAs. DsrA annealing stimulates expression of rpoS by disrupting a secondary structure in the rpoS leader, which otherwise prevents translation. Both RNAs bind Hfq with similar affinity but interact with opposite faces of the $\mathrm{Hfq}$ hexamer. Using mutations that block interactions between two of the three components, we demonstrate that $\mathrm{Hfq}$ binding to a functionally critical $(\mathrm{AAN})_{4}$ motif in $r p o S$ mRNA rescues DsrA binding to a hyperstable rpoS mutant. We also show that Hfq cannot stably bridge the RNAs. Persistent ternary complexes only form when the two RNAs are complementary. Thus, Hfq mainly acts by binding and restructuring the rpoS mRNA. However, Hfq binding to DsrA is needed for maximum annealing in vitro, indicating that transient interactions with both RNAs contribute to the regulatory mechanism.
\end{abstract}

Keywords: DsrA; rpoS; RNA chaperone; RNA-protein interactions; bacterial gene regulation; native gel electrophoresis

\section{INTRODUCTION}

Small regulatory RNAs (sRNAs) are a widespread and important part of bacterial gene regulation, mediating the cellular response to a diverse variety of external signals (Vogel and Papenfort 2006; Gottesman and Storz 2010). The major class of bacterial sRNAs regulate genes posttranscriptionally by base-pairing with complementary regions in target mRNAs to either repress or activate translation. These sRNAs require $\mathrm{Hfq}$, an abundant Sm-like protein that has several roles in bacterial RNA metabolism (Valentin-Hansen et al. 2004; Aiba 2007; Sittka et al. 2009), as a cofactor.

One important function of $\mathrm{Hfq}$ is to facilitate basepairing between sRNAs and their target mRNAs (Moller et al. 2002; Zhang et al. 2002). To understand how this occurs, we have studied how Hfq promotes interactions between DsrA sRNA and its regulatory target rpoS mRNA (Lease and Woodson 2004; Soper and Woodson 2008; Soper et al. 2010). Hfq binds many sRNAs and stabilizes

\footnotetext{
${ }^{3}$ Corresponding author.

E-mail swoodson@jhu.edu.

Article published online ahead of print. Article and publication date are at http://www.rnajournal.org/cgi/doi/10.1261/rna.2767211.
}

them against turnover (Moll et al. 2003), and specifically recognizes a $\mathrm{U}_{6}$ sequence in DsrA sRNA (Brescia et al. 2003). sRNA regulation also depends on interactions between Hfq and the target mRNA (Geissmann and Touati 2004; Soper and Woodson 2008). The rpoS mRNA leader contains an upstream $(\mathrm{AAN})_{4}$ sequence motif that binds $\mathrm{Hfq}$ tightly and is required for $\mathrm{Hfq}$ to facilitate pairing between rpoS and DsrA sRNA (Soper and Woodson 2008). The $(\mathrm{AAN})_{4}$ motif is also required for Hfq-dependent regulation of $r p o S$ translation by $r p o S$ activating sRNAs in Escherichia coli (Soper et al. 2010). Thus, Hfq can bind both the sRNA and the mRNA in this and other regulatory pairs.

Two general mechanisms have been proposed to explain Hfq's ability to facilitate base-pairing between sRNAs and mRNAs: Co-binding of two RNAs by Hfq may promote annealing by bringing the RNA strands into close proximity (Fig. 1, top). Restructuring of one or both RNAs when Hfq binds may facilitate pairing by changing the RNA secondary (or tertiary) structure (Fig. 1, bottom; Storz et al. 2004). We tested these two models by perturbing interactions among DsrA, rpoS mRNA, and Hfq.

Hfq has two RNA binding surfaces on opposite sides of the hexamer that allow it to interact with an mRNA and sRNA simultaneously (Fig. 2A; Mikulecky et al. 2004). The proximal face pore preferentially binds U-rich RNA 


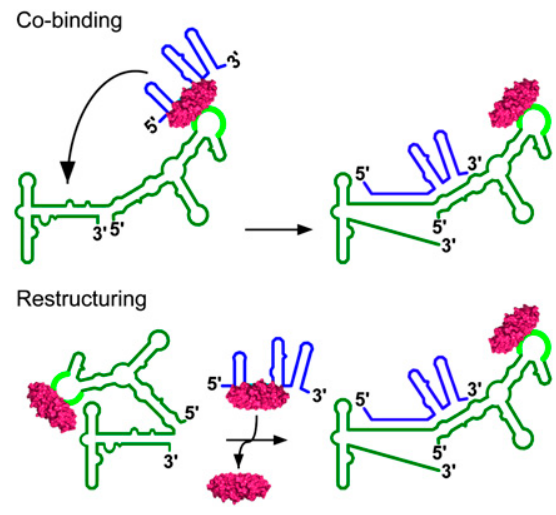

FIGURE 1. Models for Hfq facilitation of sRNA binding. Hfq (pink) may facilitate base-pairing between DsrA (blue) and the rpoS leader (green) by binding both RNAs simultaneously (co-binding; top) or by changing the structure of one or both RNAs (restructuring; bottom).

(1 nucleotide [nt] per subunit) (Schumacher et al. 2002), while the distal face binds A-rich RNA (3 nt per subunit) (Mikulecky et al. 2004; Link et al. 2009). Hfq accelerates annealing and exchange of complementary oligonucleotides (Moll et al. 2003; Arluison et al. 2007; Rajkowitsch and Schroeder 2007; Hopkins et al. 2009), in part by transiently engaging both strands in a ternary complex (Rajkowitsch and Schroeder 2007; Fender et al. 2010). Natural sRNAs and mRNAs are larger and more structured. It is not known if Hfq acts on these substrates by a similar mechanism in vivo.

Although Hfq can interact with more than one strand of RNA, there is also evidence that the chaperone activity of Hfq restructures longer RNAs and that this restructuring activity contributes to sRNA binding. Hfq was shown to induce a conformational change in the $3^{\prime}$ end of the $\mathrm{Q} \beta$ bacteriophage RNA (Schuppli et al. 1997) and change the structure of the $\operatorname{sodB}$ mRNA to facilitate its repression by the RyhB sRNA (Geissmann and Touati 2004). Chemical modification experiments showed that $\mathrm{Hfq}$ can alter or weaken the auto-inhibitory stem-loop in rpoS mRNA (Soper and Woodson 2008).

Co-binding and restructuring are not mutually exclusive, and $\mathrm{Hfq}$ may bridge the RNAs even as it restructures one or both of them. Such a combined mechanism was proposed by Arluison and $\mathrm{Ha}$ to explain how Hfq promotes the annealing of oligonucleotides designed to represent DsrA and the top and bottom strands of the rpoS inhibitory stem (Arluison et al. 2007; Hwang et al. 2011).

To differentiate between the co-binding and restructuring models of Hfq regulation, we probed which binding interactions between the rpoS mRNA leader, DsrA sRNA, and Hfq are needed to form stable ternary complexes. We previously observed that the appearance of ternary complexes correlated well with the ability of Hfq to facilitate binding of DsrA with rpoS mRNA (Soper and Woodson 2008). If the co-binding model is correct, then ternary complex formation requires both RNAs to be capable of binding Hfq, but not necessarily each other. In contrast, if $\mathrm{Hfq}$ acts by restructuring the rpoS leader, then the Hfq binding site on DsrA should be dispensable. Therefore, we created versions of the DsrA and rpoS RNAs that could only bind Hfq or each other, but not both. The results that follow show that Hfq restructures the rpoS leader to facilitate binding to DsrA. However, we also show that Hfq binding to DsrA contributes to Hfq facilitation of RNA pairing in vitro.

\section{RESULTS}

\section{Ternary complex formation requires RNA complementarity}

To test whether $\mathrm{Hfq}$ is capable of stably bridging rpoS mRNA and DsrA, we introduced mutations into DsrA sRNA designed to disrupt binding to the rpoS leader but not Hfq (DsrA RpoS-Binding-Mutant or DsrA-RBM) (Fig. $3 \mathrm{~A}, \mathrm{~B})$. These mutations were not predicted to change the DsrA secondary structure. As expected, ${ }^{32} \mathrm{P}$-labeled DsrARBM bound $\mathrm{Hfq}(\mathrm{D} \cdot \mathrm{H})$ in gel mobility shift experiments and did not bind the rpoS leader (Fig. 3C, right). However, in contrast to wild-type DsrA, DsrA-RBM did not form a stable ternary complex in the presence of both rpoS RNA and Hfq (Fig. 3C, cf. the two center lanes). These results showed that Hfq cannot stably bridge the rpoS and DsrA RNAs, despite its separate RNA binding surfaces.

\section{Hfq rescues DsrA binding to a GC-clamped rpoS leader}

To create a mutant of the rpoS leader that binds Hfq but not DsrA, we relied on a previous observation that a more stable self-inhibitory stem in the rpoS leader inhibits DsrA binding (T Soper, unpubl.). To stabilize the rpoS stem, a four


FIGURE 2. Hfq binds A-rich and U-rich RNA. (A) The crystal structure of Staphylococcus aureus $\mathrm{Hfq}$ (pale pink) bound to $5^{\prime}$ AU5G (blue) on the proximal face (Schumacher et al. 2002) was superimposed with the crystal structure of E. coli Hfq (gray) bound to A18 (green) on the distal face (Link et al. 2009). (B) Y25 (red) contributes to binding A-rich RNA in the purine-specific "R" site on the distal face. $(C) \mathrm{K} 57$ (red; K56 in E. coli) contributes to U-rich RNA binding in the proximal face pore. 
A. DsrA 5'--AUCAGAUUUCCUGGUGUAACGAAUUUUUU---3' rpoS 3'--AUGCCUAAAGGGGAACAUUGCUUAAAGUU--5' DsrA-RBM 5'--AUGUCAAAACGACGUGUCCAUAAUUUUUU--3'

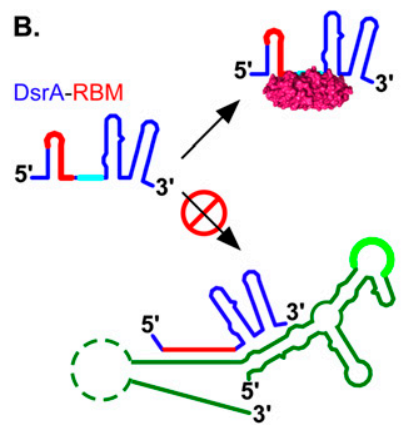

C.

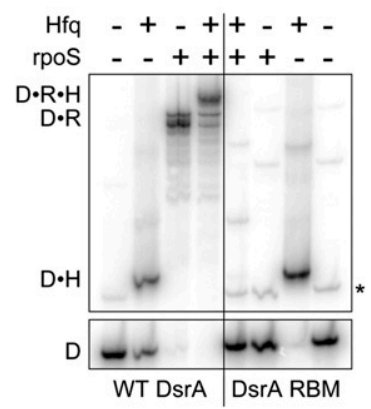

FIGURE 3. Hfq does not stably bridge DsrA and the rpoS leader. (A) Mutations in DsrA-RBM (red nucleotides) disrupt pairing to the rpoS leader (green letters). (B) The Hfq binding site of DsrA (cyan) is unchanged in DsrA-RBM, which is predicted to bind Hfq but not rpos. (C) Gel mobility shift assay, using ${ }^{32} \mathrm{P}$-labeled wild-type DsrA or DsrA-RBM (D) incubated with either $0.17 \mu \mathrm{M} \mathrm{Hfq}_{6}, 0.1 \mu \mathrm{M}$ rpoS323,

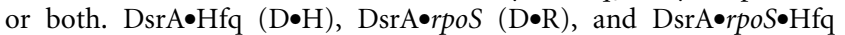
$(\mathrm{D} \bullet \mathrm{R} \bullet \mathrm{H})$ complexes are resolved with wild-type DsrA. The asterisk indicates a small amount of DsrA dimer. The blank region of the gel between $\mathrm{D}$ and $\mathrm{D} \bullet \mathrm{H}$ is not shown.

GC base-pair "clamp" was introduced into the rpoS leader (rpoS-clamp) (Fig. 4A). Since the A-rich Hfq-binding site in the upstream portion of the rpoS leader was unchanged, the rpoS-clamp RNA was predicted to bind Hfq but not DsrA.

As expected, the ${ }^{32} \mathrm{P}$-labeled rpoS-clamp formed a complex with $\mathrm{Hfq}(\mathrm{R} \cdot \mathrm{H})$ similar to wild-type $r p o S$ mRNA (Fig. $4 \mathrm{~B}$, cf. lanes 2 and 7) but did not form a complex with DsrA $(\mathrm{R} \cdot \mathrm{D})$ (Fig. 4B, cf. lanes 3 and 8). Because the experiment with DsrA-RBM showed that Hfq does not stably bridge rpoS and DsrA, we expected that the rpoS-clamp RNA would not be found in an $r p o S \bullet \mathrm{DsrA} \bullet \mathrm{Hfq}(\mathrm{R} \bullet \mathrm{D} \bullet \mathrm{H})$ ternary complex. However, in the presence of both DsrA and Hfq, about one-third of the rpoS-clamp RNA formed a ternary complex, one-third formed an rpoS•Hfq complex, and the rest remained unbound (Fig. 4B, lane 9). In contrast, nearly all wild-type rpoS mRNA formed a ternary complex under these conditions (Fig. 4B, cf. lanes 4 and 9).

To test whether the RNAs in rpoS•DsrA•Hfq ternary complexes are paired, the Hfq was competed away with polyU. For both the wild-type rpoS and rpoS-clamp, the resulting rpoS•DsrA complex was equivalent in amount to the ternary complex present before polyU treatment (Fig. $4 \mathrm{~B}$, cf. lanes 4,5 to lanes 9,10). This result indicated that Hfq partially rescues DsrA base-pairing to the rpoS-clamp mutant. However, when the $(\mathrm{AAN})_{4}$ motif was mutated in rpoS-clamp RNA, very little DsrA was bound in either the presence or absence of Hfq (Fig. 4B, lanes 13,14), supporting the conclusion that a specific interaction between $\mathrm{Hfq}$ and the rpoS leader facilitates DsrA pairing.

\section{Testing the importance of $\mathrm{Hfq}$ binding to DsrA}

If $\mathrm{Hfq}$ acts primarily by restructuring the rpoS leader via its interactions with the (AAN) $)_{4}$ repeat element, an RNA able to base-pair with the rpoS leader but incapable of binding Hfq should still behave like wild-type DsrA sRNA. To test this prediction in vitro, we used a 25-nt "mimic" of DsrA that is complementary to the top strand of the rpoS mRNA inhibitory stem but lacks an Hfq binding site (Fig. 5A).

As expected, the 25-nt DsrA mimic bound poorly to Hfq in competition experiments against wild-type DsrA (Fig. 5B). Hfq binds wild-type DsrA with a $K_{\mathrm{d}}$ of $23 \mathrm{nM}$ (Brescia et al. 2003). The DsrA mimic bound well to rpoS mRNA in gel mobility shift experiments and formed a stable ternary complex when all three molecules were present (Fig. 5C). These results raised the possibility that Hfq binding to DsrA is dispensable for its facilitation of sRNA-mRNA interactions.

To test that hypothesis further, we compared the rates by which DsrA and the 25-nt DsrA mimic bound the rpoS
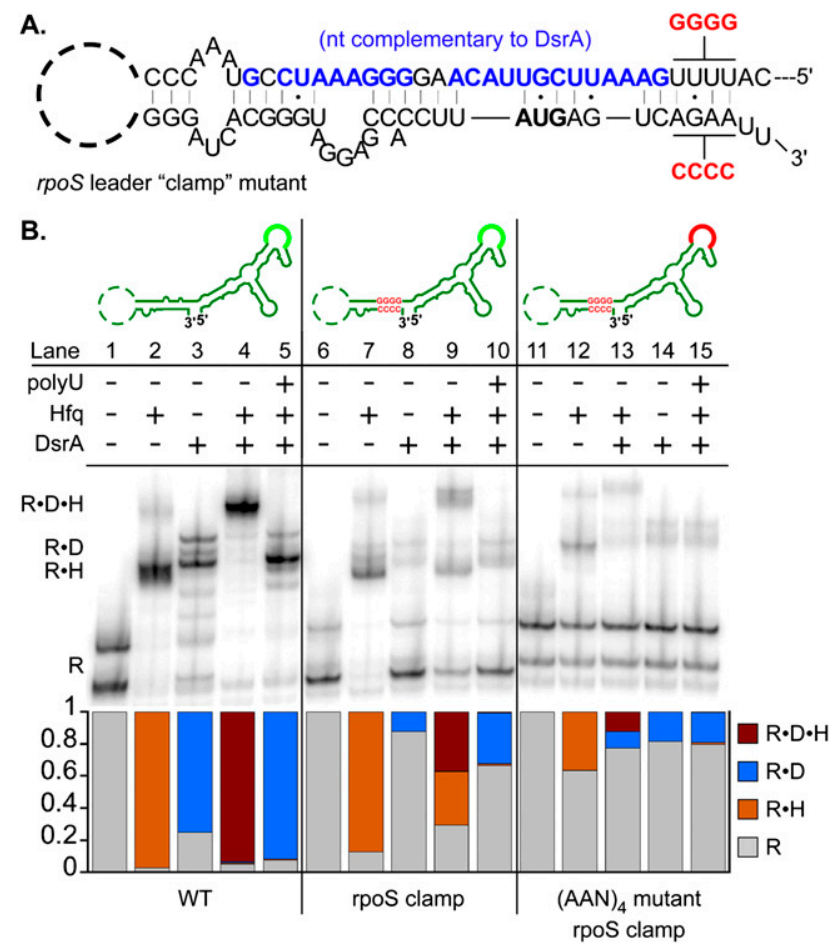

FIGURE 4. Hfq restructures a hyperstable rpoS mutant. (A) The GC clamp mutation (red) stabilizes the inhibitory stem of the $r p o S$ leader without changing its complementarity to DsrA (blue). (B) Gel mobility shift assay using uniformly ${ }^{32} \mathrm{P}$-labeled rpoS323 variants $(\sim 20 \mathrm{nM})$ in the presence of the following: $0.08 \mu \mathrm{M} \mathrm{Hfq} \mathrm{q}_{6}, 0.2 \mu \mathrm{M}$ DsrA, or both or both RNAs plus $0.8 \mathrm{mg} / \mathrm{mL}$ polyU to sequester Hfq. The rpoS323 variants were as follows: wild type (WT; lanes 1-5), rpoS-clamp (lanes 6-10), (AAN) ${ }_{4}$ mutant of rpoS-clamp (lanes 1115). The fraction of each complex is plotted beneath the gel; free

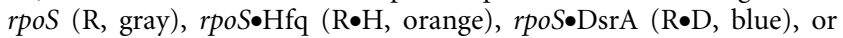
rpoS $\bullet \mathrm{DsrA} \bullet \mathrm{Hfq}(\mathrm{R} \bullet \mathrm{D} \bullet \mathrm{H}$, brown $)$. Note that in the right panel, $\mathrm{R}+\mathrm{D}+\mathrm{H}$ is loaded before $\mathrm{R}+\mathrm{D}$. 

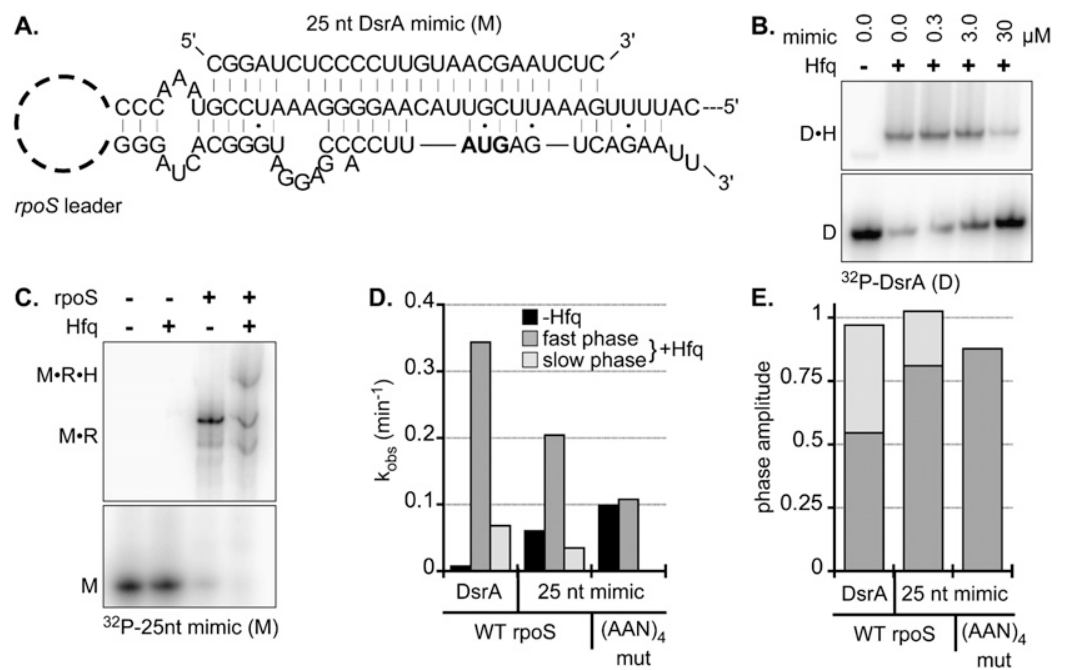

FIGURE 5. DsrA mimic bind rpoS leader but not Hfq. (A) A 25-nt DsrA mimic is complementary to the top strand of the rpoS leader but lacks an Hfq binding site. $(B)$ Competitive binding experiment with $0-30 \mu \mathrm{M}$ unlabeled $25-\mathrm{nt}$ mimic and ${ }^{32} \mathrm{P}$-labeled DsrA (D) in complex with $0.13 \mu \mathrm{M} \mathrm{Hfq}_{6}(\mathrm{D} \bullet \mathrm{H}) .(C)^{32} \mathrm{P}$-labeled 25-nt mimic $(\mathrm{M})$ in the presence of $0.13 \mu \mathrm{M} \mathrm{Hfq}{ }_{6}, 22.5 \mathrm{nM}$ rpoS323, or both. Binary mimic-rpoS complex $(\mathrm{M} \bullet \mathrm{R})$ and ternary mimic-rpoS-Hfq complex $(\mathrm{M} \bullet \mathrm{R} \bullet \mathrm{H})$ are resolved. $(D)$ Observed binding rate of DsrA or the 25-nt mimic to rpoS323; DsrA + rpoS323, left columns; mimic + rpoS 323, middle columns; mimic + rpoS323 $\Delta(\mathrm{AAN})_{4}$, right columns. Black indicates no Hfq; dark gray, fast phase in 0.13 $\mu \mathrm{M} \mathrm{Hf} \mathrm{q}_{6}$; and light gray, slow phase in $0.13 \mu \mathrm{M} \mathrm{Hf}_{6}$. (E) The amplitudes of the fast phase (dark gray) and slow phase (light gray) for Hfq-dependent binding kinetics.

leader, in the presence and absence of Hfq (Fig. 5D). The DsrA mimic bound rpoS significantly faster than did DsrA in the absence of Hfq (observed rate constants of 0.06 and $0.01 \mathrm{~min}^{-1}$, respectively) (Fig. 5D, black bars), most likely because the 25-nt RNA has no predicted self-structure to impede its interactions with the rpos leader.

Hfq greatly accelerated binding of both DsrA and the 25nt mimic to rpoS mRNA (Fig. 5D, dark gray bars). However, Hfq increased the binding rate of DsrA much more than the 25-nt mimic, indicating that direct interactions between Hfq and DsrA do contribute to its association with rpoS mRNA (Fig. 5D). This may be due in part to changes in DsrA secondary structure. Binding kinetics for wild-type $r p o S$ in the presence of Hfq were biphasic, and interestingly, the amplitude of the fast phase was larger when the mimic was used in place of DsrA (Fig. 5E), perhaps because Hfq is released from the product duplex more easily. The presence of Hfq had no effect on the observed binding rate when the (AAN) $)_{4}$ motif in rpoS was mutated (Fig. 5D), consistent with previous results indicating that $\mathrm{Hfq}$ binding to this sequence element is crucial for facilitating interactions between DsrA and the rpoS leader (Soper and Woodson 2008).

\section{Mutating the $\mathrm{A}$ and $\mathrm{U}$ binding surfaces of $\mathrm{Hfq}$}

To further explore the importance of Hfq interactions with DsrA and the rpoS leader, we asked whether Hfq mutants previously shown to disrupt binding of A-rich or U-rich nary complex in the nary complex in the presence of the two RNAs (Fig. 7A, center) and accelerated the rate of RNA complex formation (Fig. 7B). Interestingly, the rate association conferred by Hfq:K56A was less than that conferred by wild-type Hfq (3.5-fold and 10-fold, respectively), which further suggests that interactions between Hfq and DsrA play a part in Hfq chaperone activity.

We observed that Hfq:K56A bound the rpoS leader distinctly from wild-type Hfa, having weaker specific binding and fewer resolved nonspecific binding sites (Fig. 6C). This weaker binding to the rpoS leader might also account for the weaker acceleration of DsrA binding. Reduced RNA binding and chaperone activity may be also due to the lower stability of the Hfq:K56A hexamer compared with wild-type Hfq (S Panja, pers. comm.). Altogether, these data indicate that Hfq's interaction with A-rich sequences is critical for stimulating interactions between DsrA and rpoS mRNA and the formation of an $r p o S \cdot D s r A \cdot H f q$ ternary complex, while interactions between U-rich sequences and the proximal face are dispensable. These results are consistent with the results from the RNA mutants.

\section{DISCUSSION}

\section{$\mathrm{Hfq}$ restructures the rpoS leader to facilitate DsrA annealing}

How Hfq facilitates interactions between sRNAs and their regulatory targets remains an important question. We 

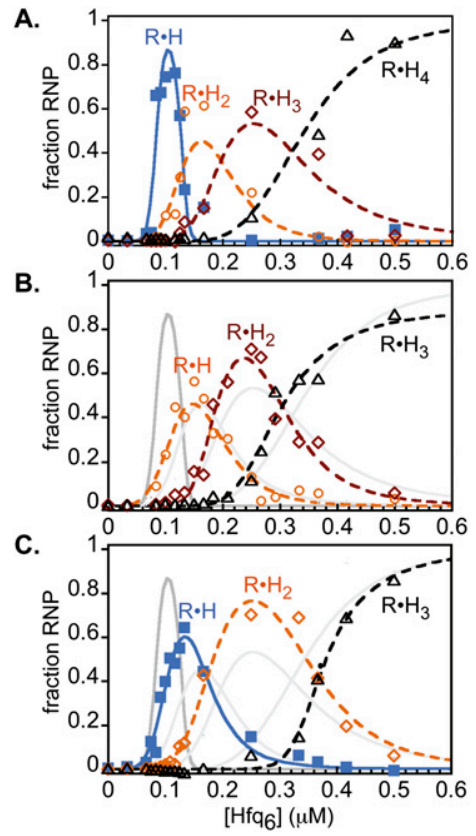

FIGURE 6. Hfq mutants binding the rpoS leader. Hfq titrations of ${ }^{32} \mathrm{P}$-rpoS323. At increasing $\left[\mathrm{Hfq}_{6}\right]$, multiple hexamers bind $r p o S$. $(A)$ Wild-type Hfq. The data are best fit with Equation 1 (Materials and Methods), which assumes one specific binding site $\left(\mathrm{R} \bullet \mathrm{H} ; \mathrm{K}_{\mathrm{s}}=0.08\right.$ $\mu \mathrm{M})$ and three equal, nonspecific binding sites $\left(\mathrm{R} \bullet \mathrm{H}_{2}, \mathrm{R} \bullet \mathrm{H}_{3}, \mathrm{R} \bullet \mathrm{H}_{4}\right.$; $\left.\mathrm{K}_{\mathrm{ns}}=0.18 \mu \mathrm{M}\right)$ on the rpoS leader. (B) Hfq:Y25D. The data are best fit with Equation 2, which assumes three equal binding sites $\left(\mathrm{R} \bullet \mathrm{H}, \mathrm{R} \bullet \mathrm{H}_{2}\right.$, $\left.\mathrm{R} \bullet \mathrm{H}_{3} ; \mathrm{K}_{\mathrm{ns}}=0.17 \mu \mathrm{M}\right)$. The Y25D data are super-imposed over the wild-type fits (light gray) to show that the nonspecific transitions overlap. (C) Hfq:K56A. The data are best fit with Equation 3, which assumes one specific binding site $\left(\mathrm{R} \bullet \mathrm{H} ; \mathrm{K}_{\mathrm{s}}=0.11 \mu \mathrm{M}\right)$, and two nonspecific binding sites $\left(\mathrm{R} \bullet \mathrm{H}_{2}, \mathrm{R} \bullet \mathrm{H}_{3} ; \mathrm{K}_{\mathrm{ns}}=0.21 \mu \mathrm{M}\right)$. The data overlap poorly with the wild-type transitions (light gray).

previously showed that Hfq binding to an A-rich motif in the rpoS leader is critical for regulation of rpoS by sRNAs (Soper et al. 2010). In this report, we conclude that this requirement for $\mathrm{Hfq}-r p o \mathrm{~S}$ binding is primarily due to restructuring of the rpoS leader when Hfq binds upstream, although its interactions with DsrA also contribute to RNA annealing.

Hfq binding is known to change the structure of some RNAs, including the $\mathrm{Q} \beta$ phage genome. The exact structural changes in rpoS leader are still under investigation. Complicating this study is the fact that the rpos leader sequences used in these experiments adopt at least two stable conformations. However, the fact that Hfq restores DsrA binding to the rpoS-clamp RNA, overcoming the increased stability of the stem, suggests that Hfq weakens the secondary structure of the rpoS leader, making it more amenable to invasion by DsrA. Hfq does not improve DsrA binding to an $(\mathrm{AAN})_{4}$ mutant version of rpoS-clamp, indicating that $\mathrm{Hfq}$ needs to interact with the leader to weaken the inhibitory stem.

This conclusion is also supported by previous chemical modification experiments (Soper and Woodson 2008), showing that Hfa makes nucleotides in the top strand of the inhibitory stem in the rpoS leader more susceptible to modification. Together these results are evidence that $\mathrm{Hfq}$ restructures the rpoS leader to facilitate DsrA binding. A similar conclusion was obtained from footprinting experiments on $\operatorname{sodB}$ mRNA (Geissmann and Touati 2004).

\section{Hfq does not stably bridge DsrA and rpoS}

Hfq is known to have separate A- and U-specific binding surfaces (Schumacher et al. 2002; Mikulecky et al. 2004; Link et al. 2009), implying that Hfq can bind the U-rich sequence in DsrA at the same time as the A-rich sequence in rpoS mRNA. However, this co-binding has not been directly demonstrated in the case of DsrA and the rpoS leader. We showed that a DsrA mutant that binds Hfq but not the rpoS leader does not form a stable $r p o S \bullet D s r A \bullet H f q$ ternary complex, indicating that Hfq cannot stably bridge DsrA and rpoS if the two RNAs are not base-paired. Further evidence that the ternary complex depends on RNA•RNA binding comes from the GC-clamped rpoS leader (rpoSclamp), which bound DsrA poorly and subsequently formed less ternary complex than the wild-type rpoS leader.

\section{Contributions from transient co-binding}

The fact that Hfq cannot stably bind both RNAs simultaneously does not preclude a role for transient co-binding of Hfq during RNA annealing. Fluorescence experiments show that Hfq binds and releases oligonucleotide substrates very rapidly (in a second or less), such that the resulting ternary complexes are short-lived (Hopkins et al. 2011). In general, the rapid dissociation of Hfq from the RNA correlates with increased annealing activity (Rajkowitsch and Schroeder 2007; Hopkins et al. 2009). Recently, Fender et al. (2010) proposed that RNAs are actively displaced from the Hfq hexamer by invasion of a second RNA strand.
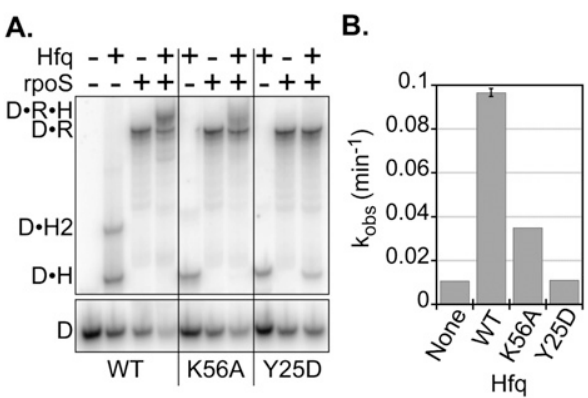

FIGURE 7. Formation of ternary complexes by Hfq mutants. (A) Gel mobility shift experiments with ${ }^{32} \mathrm{P}$-labeled DsrA (D) in the presence of $0.1 \mu \mathrm{M}$ rpoS323, $1 \mu \mathrm{M} \mathrm{Hfq}$, or both. Wild-type (WT) Hfq (left panel), Hfq K56A (center panel), and Hfq Y25D (right panel). Complexes labeled as in Figure 3. (B) Observed rate of DsrA binding to $100 \mathrm{nM}$ rpoS323 in the absence or presence of $0.17 \mu \mathrm{M} \mathrm{Hfq}$. 
By analogy with the short RNA substrates, DsrA and rpoS mRNA may interact dynamically with one or both surfaces of Hfq, catalyzing base-pairing between the two RNAs. This hypothesis raises the question of how $\mathrm{Hfq}$ interactions with the upstream $(\mathrm{AAN})_{4}$ site help recruit DsrA to its complement in the inhibitory stem-loop further downstream. One possibility is that the upstream and downstream regions are brought together by a higher-order structure in the rpoS leader. It is also possible that the $(\mathrm{AAN})_{4}$ site tethers the distal face of Hfq to the mRNA, leaving the proximal face available to engage in transient interactions with DsrA and with leader and other sequences in rpos.

\section{Interactions with DsrA sRNA}

A mechanism that combines stable interactions between Hfq and the $(\mathrm{AAN})_{4}$ motif with dynamic Hfq interactions with DsrA and downstream portions of rpoS mRNA is consistent with the observation that Hfq binding to DsrA is required for full activity. It is also consistent with the release of Hfq into solution when the RNA substrates lack an additional binding site for Hfq, recycling it for the next round of annealing or strand exchange (Lease et al. 2004). This recycling mode of action increases the annealing rate between DsrA and rpoS mRNA about two- to threefold (Lease et al. 2004), consistent with the effect of having an Hfq binding site on DsrA estimated from comparison to the 25-nt DsrA mimic (Fig. 5).

In conclusion, transient co-binding of rpoS mRNA and DsrA may help explain why Hfq binding DsrA is required for full activity and account for the ability of $\mathrm{Hfq}$ to facilitate interactions between DsrA and oligonucleotides (Hopkins et al. 2009). However, the results in this report show that co-binding is transient and insufficient for sRNA recruitment and that restructuring the rpoS leader is an important function of Hfq in sRNA regulation.

\section{MATERIALS AND METHODS}

\section{Plasmids and transcription templates}

rpoS323, rpoS323 $(\mathrm{AAN})_{4}$, and DsrA RNA were transcribed in vitro as previously described (Lease and Woodson 2004; Soper and Woodson 2008; Soper et al. 2010). The plasmid template for DsrA-RBM was constructed with two rounds of site-directed mutagenesis of pUCT7DsrA (Lease and Woodson 2004). The transcription template for rpoS323-clamp was amplified by PCR using the plasmid pRpoS576U4G4 (Soper and Woodson 2008) and a downstream primer containing complementary Cs. The plasmids for the Hfq mutants were created by site-directed mutagenesis of the plasmid pET21b-Hfq (Zhang et al. 2002).

\section{RNA preparation}

RNAs were transcribed using T7 RNA polymerase from either linearized plasmid or PCR templates and purified as previously described (Zaug et al. 1988). The synthetic 25-nt DsrA mimic (Dharmacon) was de-protected according to the supplier's specifications.

\section{Protein purification}

Hfq was overexpressed in E. coli. The Hfq used for the experiments described in Figures 3, 4, and 5 was purified with a polyASepharose column as in the methods described by Zhang et al. (2002) and Soper and Woodson (2008). The untagged Hfq used in the experiments described by Figure 6 was purified with a $\mathrm{Co}^{2+}$ column as in the method described by Soper et al. (2010). In both cases, Hfq was dialyzed against Hfq storage buffer (50 mM Tris$\mathrm{HCl}$ at $\mathrm{pH} 7.5,1 \mathrm{mM}$ EDTA, $250 \mathrm{mM} \mathrm{NH}_{4} \mathrm{Cl}, 10 \%$ glycerol by volume) and stored at $-80^{\circ} \mathrm{C}$. Hfq purified over the $\mathrm{Co}^{2+}$ column bound DsrA and the rpoS leader twofold to threefold more weakly than did Hfq purified from polyA-Sepharose. However, the relative binding affinities of different RNAs were the same.

\section{Equilibrium gel mobility shift}

As described previously, all binding reactions were performed at $25^{\circ} \mathrm{C}$ in annealing buffer $(50 \mathrm{mM}$ Tris- $\mathrm{HCl}$ at $\mathrm{pH} 7.5,250 \mathrm{mM}$ $\mathrm{NaCl}, 250 \mathrm{mM} \mathrm{KCl}$ ) and $\mathrm{Hfq}$ storage buffer, both used as $5 \times$ (Lease and Woodson 2004; Soper and Woodson 2008). For the equilibrium binding experiments examining ternary complex formation, concentrations were $\sim 20 \mathrm{nM}$ labeled RNA, $0.2 \mu \mathrm{M}$ unlabeled RNA and $0.08 \mu \mathrm{M} \mathrm{Hfq} q_{6}$, except where stated otherwise. DsrA, DsrA-RBM, and the 25-nt DsrA mimic were $5^{\prime}$-end ${ }^{32} \mathrm{P}$-labeled, while rpoS323 was uniformly ${ }^{32} \mathrm{P}$-labeled. Reactions were incubated $2 \mathrm{~h}$ at $25^{\circ} \mathrm{C}$ and resolved on a $1 \times \mathrm{TBE}$ native $6 \%$ polyacrylamide gel. The fraction of each species was calculated by dividing the number of counts in each band by the sum of counts in all the bands in the lane.

For equilibrium binding experiments with the GC-clamp mutant of rpoS323 (rpoS-clamp), half of the reaction containing rpoS323, DsrA, and Hfq was transferred to a separate tube, $1 \mu \mathrm{L}$ of $5.0 \mathrm{mg} / \mathrm{mL}$ polyU was added, and the reactions were incubated an additional $10 \mathrm{~min}$ before native gel electrophoresis.

Hfq titrations of rpoS323 were carried out and analyzed as previously described (Soper and Woodson 2008) using wild-type, $\mathrm{K} 56 \mathrm{~A}$, and Y25D Hfq purified with a $\mathrm{Co}^{2+}$ column. The specific $\left(\mathrm{K}_{\mathrm{s}}\right)$ and nonspecific $\left(\mathrm{K}_{\mathrm{ns}}\right)$ equilibrium binding constants were fit using partition functions as previously described (Lease and Woodson 2004; Soper and Woodson 2008). Wild-type Hfq was fit with Equation 1, Hfq:Y25D with Equation 2, and Hfq:K56A with Equation 3.

$$
\begin{gathered}
1+\left(\frac{[\mathrm{Hfq}]}{K_{s}}\right)^{n}+3\left(\frac{[\mathrm{Hfq}]^{2}}{K_{s} K_{n s}}\right)^{n}+3\left(\frac{[\mathrm{Hfq}]^{3}}{K_{s} K_{n s}^{2}}\right)^{n}+\left(\frac{[\mathrm{Hfq}]^{4}}{K_{s} K_{n s}^{3} L}\right)^{n} \\
1+3\left(\frac{[\mathrm{Hfq}]}{K_{n s}}\right)^{n}+3\left(\frac{[\mathrm{Hfq}]^{2}}{K_{n s}^{2}}\right)^{n}+\left(\frac{[\mathrm{Hfq}]^{3}}{K_{n s}^{3} L}\right)^{n}
\end{gathered}
$$

$$
1+\left(\frac{[\mathrm{Hfq}]}{\mathrm{K}_{\mathrm{s}}}\right)^{n}+2\left(\frac{[\mathrm{Hfq}]^{2}}{K_{s} K_{n s}}\right)^{n}+\left(\frac{[\mathrm{Hfq}]^{3}}{K_{s} K_{n s}^{2} L}\right)^{n}
$$




\section{RNA binding kinetics}

RNA annealing kinetics were performed using either $5^{\prime}$-end ${ }^{32} \mathrm{P}$ labeled DsrA or 25-nt DsrA mimic plus either rpoS323 or (AAN) ${ }_{4}$ mutant rpoS323, in the presence and absence of $0.08 \mu \mathrm{M} \mathrm{Hfq}$. Final concentrations for all RNAs were 22.5-25 nM. Reactions with the 25-nt mimic were resolved on a discontinuous $6 \% / 20 \%$ polyacrylamide gel; all other reactions were resolved on a $6 \%$ native polyacrylamide gel. Kinetic experiments with the $\mathrm{Hfq}$ mutants were performed as described previously (Soper and Woodson 2008), except that rpoS323 was present at $100 \mathrm{nM}$ and $\mathrm{Hfq}$ was present at $0.17 \mu \mathrm{M}$.

\section{ACKNOWLEDGMENTS}

We thank Dr. Julia Hopkins and Dr. Subrata Panja for providing reagents, assistance, and valuable discussions. This work was supported by a grant from the NIH (GM48846).

Received April 4, 2011; accepted May 6, 2011.

\section{REFERENCES}

Aiba H. 2007. Mechanism of RNA silencing by Hfq-binding small RNAs. Curr Opin Microbiol 10: 134-139.

Arluison V, Hohng S, Roy R, Pellegrini O, Regnier P, Ha T. 2007. Spectroscopic observation of RNA chaperone activities of Hfq in post-transcriptional regulation by a small non-coding RNA. Nucleic Acids Res 35: 999-1006.

Brescia CC, Mikulecky PJ, Feig AL, Sledjeski DD. 2003. Identification of the Hfq-binding site on DsrA RNA: Hfq binds without altering DsrA secondary structure. RNA 9: 33-43.

Fender A, Elf J, Hampel K, Zimmermann B, Wagner EG. 2010. RNAs actively cycle on the Sm-like protein Hfq. Genes Dev 24: 2621-2626.

Geissmann TA, Touati D. 2004. Hfq, a new chaperoning role: binding to messenger RNA determines access for small RNA regulator. EMBO J 23: 396-405.

Gottesman S, Storz G. 2010. Bacterial small RNA regulators: versatile roles and rapidly evolving variations. Cold Spring Harb Perspect Biol pii: cshperspect.a003798v1. doi: 10.1101/cshperspect.a003798.

Hopkins JF, Panja S, McNeil SA, Woodson SA. 2009. Effect of salt and RNA structure on annealing and strand displacement by Hfq. Nucleic Acids Res 37: 6205-6213.

Hopkins JF, Panja S, Woodson SA. 2011. Rapid binding and release of Hfq from ternary complexes during RNA annealing. Nucleic Acids Res (in press). doi: 10.1093/nar/gkr062.

Hwang W, Arluison V, Hohng S. 2011. Dynamic competition of DsrA and rpoS fragments for the proximal binding site of $\mathrm{Hfq}$ as a means for efficient annealing. Nucleic Acids Res (in press). doi: 10.1093/nar/gkr075.

Lease RA, Woodson SA. 2004. Cycling of the Sm-like protein Hfq on the DsrA small regulatory RNA. J Mol Biol 344: 1211-1223.

Lease RA, Smith D, McDonough K, Belfort M. 2004. The small noncoding DsrA RNA is an acid resistance regulator in Escherichia coli. J Bacteriol 186: 6179-6185.

Link TM, Valentin-Hansen P, Brennan RG. 2009. Structure of Escherichia coli Hfq bound to polyriboadenylate RNA. Proc Natl Acad Sci 106: 19292-19297.

Mikulecky PJ, Kaw MK, Brescia CC, Takach JC, Sledjeski DD, Feig AL. 2004. Escherichia coli $\mathrm{Hfq}$ has distinct interaction surfaces for DsrA, rpoS and poly(A) RNAs. Nat Struct Mol Biol 11: 12061214.

Moll I, Leitsch D, Steinhauser T, Blasi U. 2003. RNA chaperone activity of the Sm-like Hfq protein. EMBO Rep 4: 284-289.

Moller T, Franch T, Hojrup P, Keene DR, Bachinger HP, Brennan RG, Valentin-Hansen P. 2002. Hfq: a bacterial Sm-like protein that mediates RNA-RNA interaction. Mol Cell 9: 23-30.

Rajkowitsch L, Schroeder R. 2007. Dissecting RNA chaperone activity. RNA 13: 2053-2060.

Schumacher MA, Pearson RF, Moller T, Valentin-Hansen P, Brennan RG. 2002. Structures of the pleiotropic translational regulator Hfq and an Hfq-RNA complex: a bacterial Sm-like protein. EMBO J 21: 3546-3556.

Schuppli D, Miranda G, Tsui HC, Winkler ME, Sogo JM, Weber H. 1997. Altered 3'-terminal RNA structure in phage Q $\beta$ adapted to host factor-less Escherichia coli. Proc Natl Acad Sci 94: 10239-10242.

Sittka A, Sharma CM, Rolle K, Vogel J. 2009. Deep sequencing of Salmonella RNA associated with heterologous Hfq proteins in vivo reveals small RNAs as a major target class and identifies RNA processing phenotypes. RNA Biol 6: 266-275.

Soper TJ, Woodson SA. 2008. The rpoS mRNA leader recruits Hfq to facilitate annealing with DsrA sRNA. RNA 14: 1907-1917.

Soper T, Mandin P, Majdalani N, Gottesman S, Woodson SA. 2010. Positive regulation by small RNAs and the role of Hfq. Proc Natl Acad Sci 107: 9602-9607.

Storz G, Opdyke JA, Zhang A. 2004. Controlling mRNA stability and translation with small, noncoding RNAs. Curr Opin Microbiol 7: $140-144$.

Valentin-Hansen P, Eriksen M, Udesen C. 2004. The bacterial Sm-like protein Hfq: a key player in RNA transactions. Mol Microbiol 51: $1525-1533$

Vogel J, Papenfort K. 2006. Small non-coding RNAs and the bacterial outer membrane. Curr Opin Microbiol 9: 605-611.

Zaug AJ, Grosshans CA, Cech TR. 1988. Sequence-specific endoribonuclease activity of the Tetrahymena ribozyme: enhanced cleavage of certain oligonucleotide substrates that form mismatched ribozyme-substrate complexes. Biochemistry 27: 8924-8931.

Zhang A, Wassarman KM, Ortega J, Steven AC, Storz G. 2002. The Sm-like Hfq protein increases OxyS RNA interaction with target mRNAs. Mol Cell 9: 11-22. 

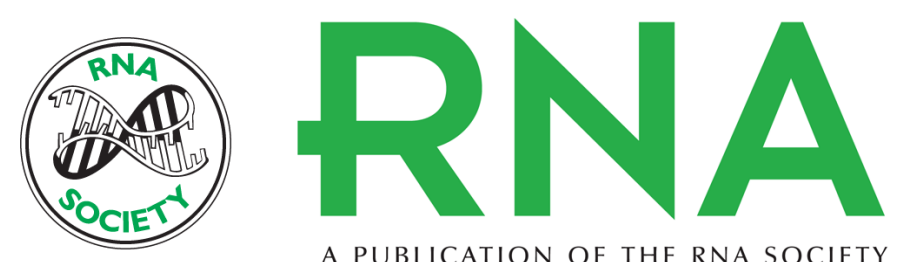

A PUBLICATION OF THE RNA SOCIETY

\section{Major role for mRNA binding and restructuring in sRNA recruitment by Hfq}

Toby J. Soper, Kevin Doxzen and Sarah A. Woodson

RNA 2011 17: 1544-1550 originally published online June 24, 2011

Access the most recent version at doi:10.1261/rna.2767211

$\begin{array}{ll}\text { References } & \begin{array}{l}\text { This article cites } 23 \text { articles, } 10 \text { of which can be accessed free at: } \\ \text { http://rnajournal.cshlp.org/content/17/8/1544.full.html\#ref-list-1 }\end{array}\end{array}$

License

Email Alerting Receive free email alerts when new articles cite this article - sign up in the box at the Service top right corner of the article or click here.

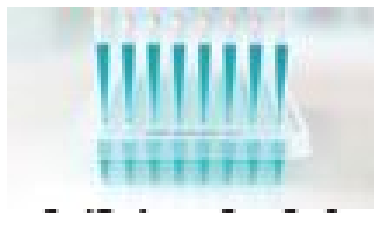

